\title{
Les manuscrits bilingues de Beckett
}

La combinaison des approches « documentaire » et « textuelle » dans une édition numérique

Dirk van Hulle

\section{OpenEdition}

Journals

Édition électronique

URL : http://journals.openedition.org/recherchestravaux/87

DOI : $10.4000 /$ recherchestravaux.87

ISSN : 1969-6434

Éditeur

UGA Éditions/Université Grenoble Alpes

Édition imprimée

Date de publication : 15 juin 2008

Pagination : $53-58$

ISBN : 978-2-84310-125-0

ISSN : 0151-1874

Référence électronique

Dirk van Hulle, "Les manuscrits bilingues de Beckett », Recherches \& Travaux [En ligne], 72 | 2008, mis en ligne le 15 décembre 2009, consulté le 03 février 2021. URL : http://journals.openedition.org/ recherchestravaux/87 ; DOI : https://doi.org/10.4000/recherchestravaux.87 
Dirk VAn Hulle

Université d'Anvers

\section{Les manuscrits bilingues de Beckett : la combinaison des approches «documentaire» et «textuelle» dans une édition numérique*}

Le sous-titre du colloque - «L'apport et les limites du numérique»contient ce qui, en traductologie, est désigné comme un «faux-ami» : dans le contexte de l'édition numérique, le mot français «numérique» ne traduit pas l'anglais «numerical» mais «digital». Cependant, dans cette contribution, je voudrais aborder une question véritablement «numérique» (numerical), car cela me semble de nature à rendre service pour l'édition et la valorisation des manuscrits.

D’un côté, des projets comme HyperNietzsche ${ }^{1}$ sont centrés sur les images. Ce sont en effet les fac-similés (ou des reproductions scannées) des documents qui constituent le point de départ. Le grand avantage de cette approche «documentaire» (basée sur l'image du document) est qu'elle fonctionne avec des cotes uniques et stables qui identifient chacune une zone particulière d'un document particulier. D'autre part, il existe une approche «textuelle» (centrée sur le texte du manuscrit), qui prend pour point de départ des transcriptions encodées en XML. Mon propos sera d'examiner si ces deux approches s'excluent mutuellement, ou si elles peuvent se combiner.

* Traduction de l'anglais par Fr. Leriche, revue par D. Van Hulle.

I. P. D’Iorio et al., HyperNietzsche: www.hypernietzsche.org. Voir la présentation de cette plateforme dans le présent numéro, p. 279. 


\section{Fac-similés et transcriptions, le topographique et le linéaire}

Dans bien des cas, il peut être utile d'appliquer à la fois une approche «documentaire» et une approche «textuelle». Par exemple, dans le cas du cahier MS $4747 \mathrm{Ib}$ de James Joyce (conservé à la British Library)² - qui montre quelques analogies avec les cahiers de Marcel Proust - il parait utile de combiner les deux. Joyce utilisa ce cahier pour rédiger plusieurs brouillons de différents chapitres de son dernier ouvrage, Finnegans Wake. Ce cahier est le témoin matériel de l'une des phases les plus créatives dans le processus d'écriture de Finnegans Wake. Il contient plusieurs sections en provenance de six chapitres. Mais ces sections ne se suivent pas nettement dans l'ordre chronologique de leur composition. Joyce a commencé avec l'intention d'écrire seulement sur la page de droite, n'utilisant les versos que pour de brèves additions, mais après un certain temps, les différentes sections se sont trouvées enchevêtrées et il semble que cet enchevêtrement a eu un effet de stimulation sur l'écriture. Aussi a-t-il commencé à remplir les pages où il trouvait des espaces blancs, écrivant même quelquefois à l'envers.

Par conséquent, en plus d'une visualisation page à page de la transcription, une visualisation section par section était également utile. Pour reconstituer la dynamique de ce processus d'écriture, il nous fallait un outil pouvant nous aider à reconstituer la chronologie des différentes sections. Pour cela, une transcription linéaire en XML, «textuelle», s'est avérée pertinente. Jean-Louis Lebrave s'est fait l'avocat des transcriptions «diplomatiques» ou topographiques, au motif que les transcriptions linéaires réduisent le manuscrit à un modèle textuel' ${ }^{3}$. Mais, si l'édition fournit des reproductions et des transcriptions topographiques, une transcription linéaire peut constituer un apport supplémentaire, justement parce qu'elle réduit le manuscrit à un modèle textuel.

Cette démarche s'est avérée utile pour étudier la genèse des derniers textes de Samuel Beckett, notamment le cahier connu sous le nom de «Super Conquérant» (Reading University Library MS 2934), dans lequel Samuel Beckett a écrit la plupart des brouillons de son avant-dernier ouvrage, Stirrings Still /Soubresautst. Une représentation qui met le document au centre part des reproductions et/ou de ce qui s'en rapproche le plus : les transcriptions

2. J. Joyce The James Joyce Archive, sous la direction de M. Groden, avec la collaboration de H.-W. Gabler, D. Hayman, A. Walton Litz, et D. Rose, New York-Londres, Garland, I 979.

3. J.-L. Lebrave, «L'édition génétique», Les Manuscrits des écrivains, A. Cadiot et Ch. Haffner éd., CNRS éditions/Hachette, I 993, p. 2 I 4.

4. S. Beckett, manuscrits de Stirrings Still, RUL MS 2933-35, 2859 , 3543, 3559, 3405, Beckett International Foundation, Reading University Library (1983-1989). 
topographiques (diplomatiques). Ce cahier contient plusieurs versions de différentes sections de cet ouvrage, mais entre-temps, tout en écrivant dans ce cahier, Beckett faisait aussi des brouillons et des dactylographies sur des feuilles volantes. Pour reconstituer la dynamique de ce processus d'écriture, il fallait permettre à l'utilisateur de comparer différentes versions. Pour cela, une transcription linéaire, encodée en XML, est d'une grande utilité. Pour comparer les versions, plusieurs options se présentent. Certains utilisateurs préfèrent comparer de larges sections en même temps, d'autres préfèrent se focaliser sur un niveau plus restreint. D'où la possibilité que nous avons offerte de comparer les versions selon des unités de dimension différente : large, moyenne, petite, c'est-à-dire la section, le paragraphe, et la phrase, en utilisant les balises $<\operatorname{div}>,<p>$ et $<$ seg $>$. Dans quelques-uns de ses ouvrages expérimentaux, Beckett n'écrit pas de phrases complètes, de sorte qu'il devient difficile de définir ce à quoi la balise $<$ seg $>$ doit correspondre. Par exemple Pas moi (Not I) est un flux de mots et de fragments de phrases coulant d'une Bouche, et interrompu seulement par des ellipses. Il n'y a ni sections, ni paragraphes, ni phrases; nous avons donc eu recours à la subdivision établie par Beckett dans son propre Synopsis (RUL MS I 227-7-I 2-IO) pour diviser le texte en segments ou «lexies».

L'histoire de la composition d'une phrase peut être visualisée avec la liste de toutes ses versions dans l'ordre chronologique. L'option «variantes génétiques» met en lumière les «réécritures» entre les différentes versions. Ceci est possible grâce aux aspects «numériques» sous-jacents de l'encodage XML. Pour qu'on puisse comparer une même phrase dans chacune des versions, il nous a fallu les numéroter. Si l'on veut combiner l'approche documentaire et l'approche textuelle, il est utile de numéroter les segments de deux façons, en utilisant les attributs de la balise $<\mathrm{seg}>$. Pour la numérotation documentaire, nous utilisons l'attribut «id». Cet «id» est le numéro de la phrase telle qu'elle apparaît sur le document, ce qui peut se faire selon le modèle proposé par le projet HyperNietzsche. Par exemple, <seg id="BEC-Sts-MS 2934, I or[I 4]"> désigne «Beckett, Stirrings Still, manuscrit 2934, page Io recto, phrase zone I4». C'est un identifiant unique, dans la mesure où il ne peut y avoir qu'un seul numéro de zone I 4 sur la page io recto du cahier «Super Conquérant».

En plus de cette numérotation documentaire, on établit une numérotation textuelle en utilisant un attribut «n». Cette numérotation lie le segment à la phrase correspondante (ou au passage correspondant) dans le texte de base. Si la phrase (ou le passage) de la page ıo recto zone $\mathrm{I} 4$ correspond, disons, à la I $27^{\mathrm{e}}$ phrase du texte de base, l'attribut «n» sera <seg n="BEC-StsMS 2934,[0 [ 27]">. De la sorte, on peut appeler toutes les balises <seg> dotées du même attribut «n» pour comparer toutes les versions de cette 
phrase. Un attribut «version» (un ajout spécifique à la DTD appliquant le codage de la $\mathrm{TEI}^{\text {s}}$ ) peut être utilisé pour indiquer le numéro de la version, par exemple version $=\ll 05 »$.

\section{Les paralipomènes et la comparaison des versions}

Évidemment, il n'est pas toujours possible de suivre l'histoire de chaque phrase de manière aussi nette. Des auteurs différents ont des habitudes différentes d'écrire. Certaines notes peuvent ne jamais parvenir jusqu'à la version finale, d'autres n'appartiennent même à aucune version. On les désigne habituellement comme "paralipomènes». Un bon exemple dans la genèse de Stirrings Still de Beckett : la note "per lungo silenzio fioco», qui se trouve dans le cahier 2934, à gauche, page 9 verso. Pour être exact, cette note n'appartient pas à la version du texte du recto qui lui fait face, mais entretient pourtant un rapport avec ce texte. Sur la page de droite, Beckett a écrit une phrase contenant le mot «faint» (faible, évanescent). Cet adjectif a dû lui rappeler un passage de l'Enfer de Dante, chant I, où Dante est abordé par une vague figure qui, plus tard seulement, s'avère être Virgile. Cette figure semble affaiblie (faint) ou enrouée à la suite d'un long silence (hoarse from long silence) - "per lungo silenzio fioco». Beckett a jeté cette note sur la page de gauche, et essayé de la traduire (en hésitant entre «faint» et «hoarse» comme équivalents de «fioco»). Il a envisagé de remplacer l'adjectif «faint» par «boarse from long silence». Mais finalement, il l'a remplacé de nouveau par «faint». Ainsi, en apparence, rien ne semble avoir changé entre la première couche et la couche finale du manuscrit. Dans le texte publié, il est peu probable que quiconque soupçonne la présence de Dante. C'est seulement grâce aux manuscrits que cet hypotexte dantesque devient explicite.

Traditionnellement, la note «per lungo silenzio fioco» ne fait pas partie de l'apparat critique d'une édition savante, car elle n'appartient à aucune version. Dans le «Beckett Digital Manuscript Project ${ }^{6} »$, elle est transcrite et par conséquent, on peut la trouver parmi les transcriptions intégrales, mais parce qu'elle n'appartient à aucune version, elle n'apparait pas dans l'apparat critique. Peut-être faudrait-il prévoir cette option.

5. Tandis que la TEI-Ps (la version la plus récente de la Text Encoding Initiative) se développe constamment, des besoins spécifiques se présentent de temps en temps et nécessitent parfois un ajout. Chaque ajout est documenté dans la DTD.

6. Ce projet est une collaboration entre la Beckett International Foundation (Université de Reading) et le Centre for Manuscript Genetics (Université d'Anvers). 
Une façon de créer cette option serait d'ajouter un attribut supplémentaire, par exemple l'attribut «type», indiquant si la phrase appartient à une version ou aux paralipomènes. La phrase «Pause then [...] and then again faint from far within oh how \& here that missing word it were to end where never till then» pourrait être encodée comme suit :

<seg id="BEC-Sts-MS 2934, Ior[14]" n="BEC-Sts-MS2934,[or 27]" type="version" version="og" $>$ Pause then $[\ldots .$.$] and then again faint from far within oh$ how \& here that missing word it were to end where never till then. $</$ seg $>$

La note sur le verso en face aurait le même attribut «n» (parce qu'elle se rapporte à cette même phrase), mais accompagné d'un attribut «type» «paralipomena» au lieu de «version» :

<seg id="BEC-Sts-MS 2934,09v[02]" n="BEC-Sts-MS 2934,[or 27]" type="paralipomena" version=" "09">per lungo silenzio fioco / faint / hoarse from long silence $</$ seg $>$

Dans l'apparat critique, nous pouvons ainsi créer la possibilité de visualiser I) ou bien les versions du texte seulement, comme dans un apparat critique traditionnel; 2) ou bien les versions du texte et les paralipomènes correspondants.

Cela peut sembler inutile, mais il y a des cas où les paralipomènes peuvent indiquer des changements d'intentions qui ont des conséquences interprétatives sérieuses. Dans un cas comme celui de la référence à Dante, il semble important de créer l'option d'intégrer de tels paralipomènes dans une vue synoptique de la phrase. L'avantage qu'il y a à encoder et à numéroter les paralipomènes, c'est qu'ils peuvent facilement être liés à la bibliothèque de l'écrivain, par exemple. Dans les exemplaires de la Divine Comédie de Beckett, le vers en question de l'Enfer, chant I ("«per lungo silenzio parea fioco») peut bien sûr être lié à une reproduction de la page de gauche du cahier RUL MS 2934, au moyen d'une numérisation du document. Mais grâce à la numérotation, il est possible de lier l'approche «documentaire» (basée sur l'image) à une approche «textuelle».

L'identifiant «id», établi à partir du document, est une cote fixe, unique. L'attribut «n» n'est pas nécessairement unique, car le numéro [o I 27] renvoie au numéro de la phrase dans le texte de base. Ce numéro se combine avec la cote du manuscrit (MS 2934), mais comme ce manuscrit peut contenir plus d'une version du texte, il se peut qu'il contienne deux versions (ou davantage) de cette phrase I 27. Si l'attribut «n» est combiné avec l'attribut «type» et l'attribut «version» (indiquant le numéro de la version), il devient également un identifiant unique, «textuel», solution alternative à l'identifiant «documentaire» unique.

Ce pourrait être le moyen d'aplanir le passage entre les approches «documentaire» et «textuelle», d'intégrer les paralipomènes dans l'édition, de les lier 
à des sources externes, et d'indiquer leur place dans la dimension temporelle du processus d'écriture. Un tel système de numérotation permet de cartographier non seulement les références intertextuelles à des sources externes, mais encore les références «intratextuelles» entre les différents textes au sein de l'œuvre de Beckett lui-même, en commençant par les auto-traductions. Quand Beckett a traduit «faint» en français, il a remplacé l'adjectif par l'expression «à peine un murmure». Dans l'édition', l'utilisateur peut comparer chaque version avec n'importe laquelle des autres versions, mettant en lumière non seulement les variantes génétiques, mais aussi les variantes de traduction. Les versions anglaises et françaises sont traitées de la même façon, de sorte que le processus d'auto-traduction est présenté comme appartenant à la genèse. Dans ce cas particulier, la légère variation dans la traduction «à peine un murmure» fait écho à tout un historique d'allusions et de voix dans les ouvrages précédents de Beckett. Par exemple, on peut la relier aux feuilles d'En attendant Godot, et aux fameuses «voix mortes» qui «murmurent», selon Estragon : «Elles murmurent» ${ }^{8}$. Rétrospectivement, sachant que «à peine un murmure» est la traduction par Beckett de «faint», qui est directement lié au vers de Dante "per lungo silenzio fioco», il est possible de rendre ces liens explicites. Le texte publié d'En attendant Godot ne suggère pas explicitement de référence à Dante, mais la combinaison «intratextuelle» des voix mortes et du murmure suggère un commun hypotexte dantesque. Grâce aux paralipomènes des manuscrits de Stirrings Still, écrit environ quarante ans après En attendant Godot et dix ans après la mise en scène de cette pièce par Beckett lui-même au théâtre Schiller de Berlin, il devient clair que les murmures d'En attendant Godot, associés aux voix mortes, sont liés à la scène cruciale de l'Enfer de Dante où Virgile apparaît pour la première fois, «per lungo silenzio fioco».

Pour conclure : afin de valoriser les manuscrits de l'œuvre de Beckett, l'un des apports majeurs des moyens informatiques est, à mon avis, la possibilité de rendre le matériau textuel tellement flexible qu'on puisse aisément mettre en évidence les liens intertextuels et intratextuels. Une transcription linéaire encodée en XML n'est pas nécessairement une réduction à un modèle textuel; elle peut rendre le matériau textuel assez flexible pour qu'on étudie chaque phrase de l'avant-texte non pas seulement dans son environnement topographique, documentaire, mais aussi dans le cadre de la structure synchronique du récit, et dans l'axe diachronique de l'avant-texte. C'est possible grâce à la numérotation. Dans ce sens, «l'apport du numérique» est une question non seulement de numérisation mais aussi de numérotation. 